\title{
SOP beim akuten Koronarsyndrom - Update 2018
}

Martin Möckel

\author{
In den Leitlinien zum akuten Koronarsyndrom (ACS) sind die Behandlungsoptionen, \\ aber auch die Notwendigkeit der Netzwerkbildung zwischen den Bereichen \\ der Versorgung festgeschrieben. Wir beschäftigen uns in diesem Beitrag mit \\ der Umsetzung der Leitlinien in krankenhausbezogene SOP am Beispiel des \\ ST-Hebungsinfarkts (STEMI).
}

\section{Einleitung}

$\begin{array}{ll}\text { ABKÜRZUNGEN } \\ \text { ACS } & \text { akutes Koronarsyndrom } \\ \text { DTW } & \text { Diagnosis-to-Wire } \\ \text { eEPK } & \text { (ereignis)gesteuerte Prozesskette } \\ \text { PCI } & \text { perkutane Koronarintervention } \\ \text { NSTEMI } & \text { Nicht-ST-Hebungs-Infarkt } \\ \text { STEMI } & \text { ST-Hebungs-Infarkt }\end{array}$

Die traditionelle Auffassung der medizinischen Diagnostik und Therapie sieht den individuellen Arzt in seinem Heilversuch am einzelnen Patienten. Dies behält zwar im Kern der Arzt-Patienten-Beziehung seine Richtigkeit. Mit der Zunahme der Komplexität moderner Heilverfahren wird die erfolgreiche Behandlung jedoch zunehmend von einem größeren und oft sektoren- und berufsgruppenübergreifenden Team realisiert.

Die moderne Medizin stellt den einzelnen Arzt, aber auch die verantwortlichen Manager im Gesundheitswesen (Klinikleitungen, Verantwortliche im Rettungsdienst, Gesundheitspolitiker usw.) vor neue Herausforderungen. Ging es lange Zeit vorwiegend um die Entwicklung innovativer neuer Heilverfahren, so stellt sich heute zunehmend die Aufgabe, diese Verfahren bezahlbar und effektiv möglichst vielen Menschen nutzbar zu machen.

Das akute Koronarsyndrom mit seinen zahlreichen und komplexen Ausprägungen ist ein gutes Beispiel für die Möglichkeit und Notwendigkeit eines standardisierten Vorgehens zum Nutzen der Patienten, des Behandlungsteams aus Ärzten, Pflege, Rettungsdienst und anderen sowie des Gesundheitswesens allgemein. Der Grund dafür ist, wie einerseits in der „Third universal Definition“ des Myokardinfarkts [1] deutlich wird, dass sich das Verständnis dieser häufigen Erkrankung einem immer schneller werdenden Wandel unterzieht. Dabei hat die Infarktforschung die Dokumentations- (ICD) und Abrechnungssysteme, mitunter aber auch Teile des Gesundheitswesens weit hinter sich gelassen. Zugleich hat die Therapie mit der Verbreitung der akuten perkutanen Koronarintervention (PCI) und zusätzlicher medikamentöser Begleittherapien wie beispielsweise der verbesserten Plättchenhemmung eine früher nie für möglich gehaltene Effektivität und Reduktion der Sterblichkeit am akuten Myokardinfarkt erreicht. Dies gilt allerdings nur dann, wenn bei jeder Gelegenheit diese Therapien genutzt und so angewendet werden, wie dies in Studien untersucht und belegt wurde. In einer Reihe von Publikationen [26] hat die Task Force Klinische Behandlungspfade der Kommission für Klinische Kardiologie der DGK (jetzt: Medical Apps in der Kardiologie) sowohl die Methodik einer standardisierten Aufbereitung als auch Beispiele der praktischen Umsetzung anhand des STEMI, NSTEMI, der antithrombotischen Therapie und des Komplikationsmanagements im Herzkatheter dargestellt.

Die Vorhaltung von Checklisten entlang einer übersichtlichen Abbildung des jeweiligen Behandlungsablaufs in Flowcharts dient dabei in erster Linie der (Patienten)Sicherheit, in zweiter Linie dem individuellen Behandlungserfolg und schließlich der Ermittlung von Kennzahlen für das Qualitätsmanagement. Zukünftig werden intuitiv bedienbare IT-Systeme, z.B. eine „CPU-App“, die Vernetzung und das Zusammenspiel der Teams erleichtern und die Optimierung der individualisierten Therapie unterstützen.

Im Folgenden werden diese Themen zusammenfassend dargestellt und erläutert. 


\section{Methodische Grundlagen: die Umsetzung von Leitlinien in Ereignisprozessketten}

Die grundlegenden Ideen zur standardisierten Reorganisation medizinischer Abläufe werden aus den Wirtschaftswissenschaften entlehnt. Obwohl dort „Geschäftsprozesse“ im Fokus stehen, die nicht ohne Weiteres mit medizinischen Prozessen vergleichbar sind, können doch wertvolle Anregungen gewonnen und nützlich umgesetzt werden. Dabei steht der Gedanke einer prozessualen im Unterscheid zu einer funktionalen Organisation im Mittelpunkt:

- In diesem Sinne stellt die Medizin heute noch vielfach eine funktionale Organisation dar, befindet sich aber in einem schneller werdenden Wandel, der vor allem auch ökonomisch und durch die Bedürfnisse der Patienten getrieben wird. Die funktionale Organisation ist durch Hierarchien sowie komplexe Planungs- und Steuerungssysteme gekennzeichnet. Nicht die Kunden (Patienten), sondern die Verrichtungen (z. B. medizinische Funktionen) bestimmen das Handeln. Dies wird in der Kardiologie besonders deutlich und leitet sich historisch aus den hohen Anforderungen der technischen Leistungen (z. B. im Herzkatheterlabor) und deren Risiken ab.

- Im Gegensatz dazu ermöglicht die prozessuale Organisation ein flexibles und schnelles Reagieren auf geänderte Anforderungen und Umfeldbedingungen. Die gemeinsame Sicht (z. B. der medizinischen Fachdisziplinen) auf die Kunden (hier Patienten) bestimmt das Handeln.

Aufgrund der Besonderheit und herausragenden Verantwortung, die im Kern der Arzt-Patienten-Beziehung liegt, ist jedoch der unumgängliche Wandel zur prozessualen Organisation im Gesundheitswesen besonders schwierig und herausfordernd.

Ein erster wesentlicher Schritt ist dabei die Herstellung einer gemeinsamen Sicht auf den sektorenübergreifenden Behandlungsprozess (z. B. am Beispiel des STEMI), in dem dann im zweiten Schritt Aufgaben und Verantwortlichkeiten flexibel verteilt werden können. Die gemeinsame Sicht erfordert eine weitgehende Abstraktion unter Berücksichtigung der Leitlinien, darf jedoch nicht so allgemein gehalten sein, dass sie beliebig wäre. Unserer Einschätzung nach ist die Darstellung der jeweiligen Leitlinie als erweiterte ereignis(gesteuerte) Prozesskette (eEPK) der beste Weg zu einer prozessorientierten Sichtweise mit potenziell vielfältigen Möglichkeiten, die Leitlinien in nachfolgenden Schritten in IT-Anwendungen einzubinden.

Exemplarisch wurde die Leitlinie zum Vorgehen beim STEMI in eine eEPK umgesetzt. Bei der Erstellung der
eEPKs haben wir uns aus Gründen der Übersichtlichkeit dafür entschieden, zusätzlich zur Definition der Elemente durch ihre Form eine farbliche Gestaltung zu benutzen. Dies folgt dem Ampelprinzip mit Darstellung von:

- Ereignissen rot,

- Funktionen grün,

- Organisationseinheiten gelb,

- Informationsobjekte orange,

- Prozesswegweiser durch ihren weißen Grund,

- Konnektoren, Flüsse und Zuordnungen schwarz-weiß.

Die wesentlichen beiden Ablaufelemente Ereignis (rot) und Funktion (grün) können so auch bei komplexen eEPKs schnell nachvollzogen werden ( $\bullet$ Abb. 1).

Weitere Details und wissenschaftliche Hintergründe der Prozessmodellierung finden sich bei Vollert u. Mitarb. [2].

\section{STEMI: sektorenübergreifendes standardisiertes Vorgehen}

Der STEMI weist eine Reihe von Kriterien auf, die sehr stark für ein standardisiertes Vorgehen sprechen [3]. So wird auch in den aktuellen Guidelines der ESC [7] vermehrt darauf hingewiesen, dass Infarktnetzwerke zu organisieren wären und die Vorgaben zu erlaubten Verzögerungszeiten, im Mittelpunkt die neu definierte „Diagnosis-to-Wire“-(DTW-)Zeit, sind ehrgeizig vorgegeben und nur durch reibungslose Zusammenarbeit zu realisieren.

\section{Merke \\ Die Abschaffung der jahrzehntelang etablierten „Door-to-Balloon“-Zeit spiegelt die stärker prozes- suale Sicht der Guidelines und die Anpassung an den technischen Fortschritt wider. Der Prozess zur Einleitung einer Reperfusionstherapie kann ja erst mit der Diagnose des STEMI beginnen.}

Der jetzt abgetrennte Prozess bis zur Diagnose liegt oft in getrennter Verantwortung und hat eigene Determinanten. Schließlich erfolgt die Reperfusion häufig bereits mit dem Vorbringen des koronaren Führungsdrahts („Wire“) und die Therapie ist dann nicht immer der Ballon, sondern ggf. ein direktes Stenting oder in einzelnen Fällen eine Thrombusaspiration.

\section{Merke}

Die DTW-Zeit ist der neue „Key Performance Indicator“ für das Qualitätsmanagement der katheterbasierten Reperfusionstherapie beim STEMI [7].

Es konnte bereits gezeigt werden, dass z. B. Feedbacksysteme einen deutlichen Nutzen für die Beschleunigung von Abläufen haben [8]. Für den innerklinischen Gebrauch ist es hilfreich, wenn die grundlegende und für 


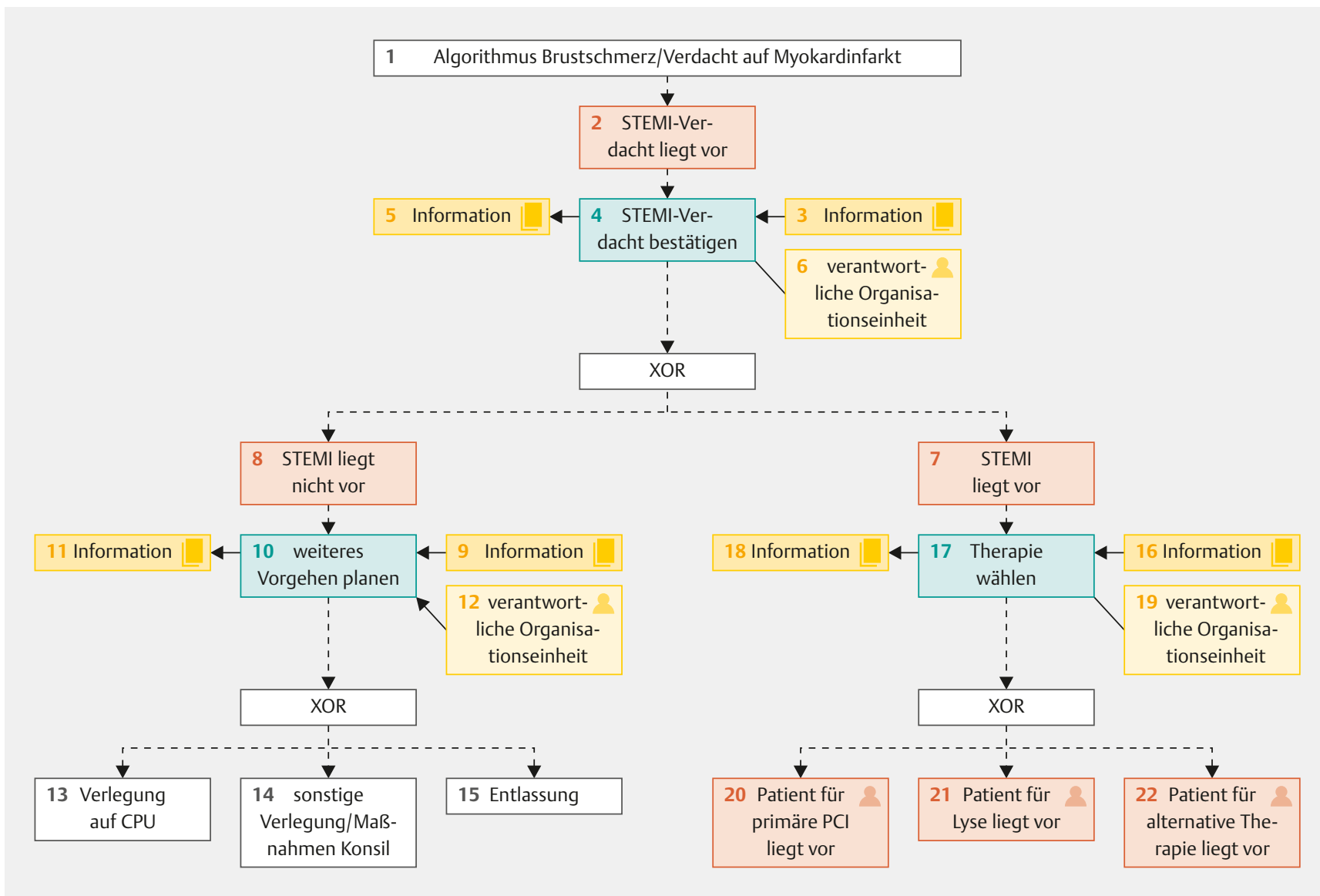

- Abb. 1 Auszug aus der eEPK des STEMI mit Erläuterungen zur Notation. Kriterien zur EKG-Ableitung und Beurteilung werden als „Standard operating Procedure“ (SOP) z. B. in Form eine Checkliste hinterlegt [2]. [rerif]

das konkrete Umfeld spezifizierte Verfahrensweise in einem Flowchart niedergelegt ist, das eine schnelle Orientierung erlaubt und Hinweise auf Checklisten, Dosierungstabellen und andere nützliche Informationen enthält. Dabei stellt die oben erwähnte eEPK den allgemeingültigen Konsens dar, während in dem lokalen Flowchart konkrete Festlegungen bei alternativen Möglichkeiten erfolgen (z. B. Auswahl des ADP-Antagonisten in der antithrombozytären Therapie, wo Ticagrelor, Prasugrel und Clopidogrel zur Verfügung stehen). Zukünftig werden diese Informationen IT-basiert und mit dem Klinikinformationssystem vernetzt auf mobilen Endgeräten noch effektiver den Prozess unterstützen. - Abb. 2 zeigt beispielhaft ein Flowchart zur innerklinischen Prozessunterstützung beim STEMI mit entsprechenden Querverweisen.

\section{NSTEMI: standardisierte Lösungen bei komplexen Krankheitsbildern}

Anders als beim STEMI liegen dem NSTEMI eine Reihe verschiedener Pathophysiologien zugrunde, die nicht alle durch katheterbasierte Therapien behandelbar sind. So wird in der universellen Infarktdefinition der Begriff für ischämisch bedingten Myokardzelluntergang reserviert, der nicht immer auf einem nicht okklusiven, rupturierten, koronaren Plaque beruht [1]. Umso drängender stellt sich die Frage, welche antithrombotischen Konzepte verfolgt werden und zu welchem Zeitpunkt eine Koronarangiografie erfolgen soll. Dabei muss im Sinn behalten werden, dass auch Patienten mit NSTEMI komplette Gefäßverschlüsse aufweisen können und entsprechend gefährdet sind. In einer Arbeit zum infarktbedingten kardiogenen Schock hatten etwa 30\% dieser Patienten die Diagnose NSTEMI [9]!

Das standardisierte Vorgehen beim NSTEMI bezieht sich wesentlich auf die zeitnahe Erfassung zusätzlicher Variablen, insbesondere Biomarker, und die zeitgerechte und wiederholte Risikostratifizierung. Details des Vorgehens und entsprechende Standards sind bei Moser u. Mitarb. [5] ausführlich dargestellt worden. Dabei ist es wie immer möglich, z. B. die Risikostratifizierung mit unterschiedlichen Scores vorzunehmen (GRACE- versus TIMI- oder HEART-Score). 


\section{STEMI}

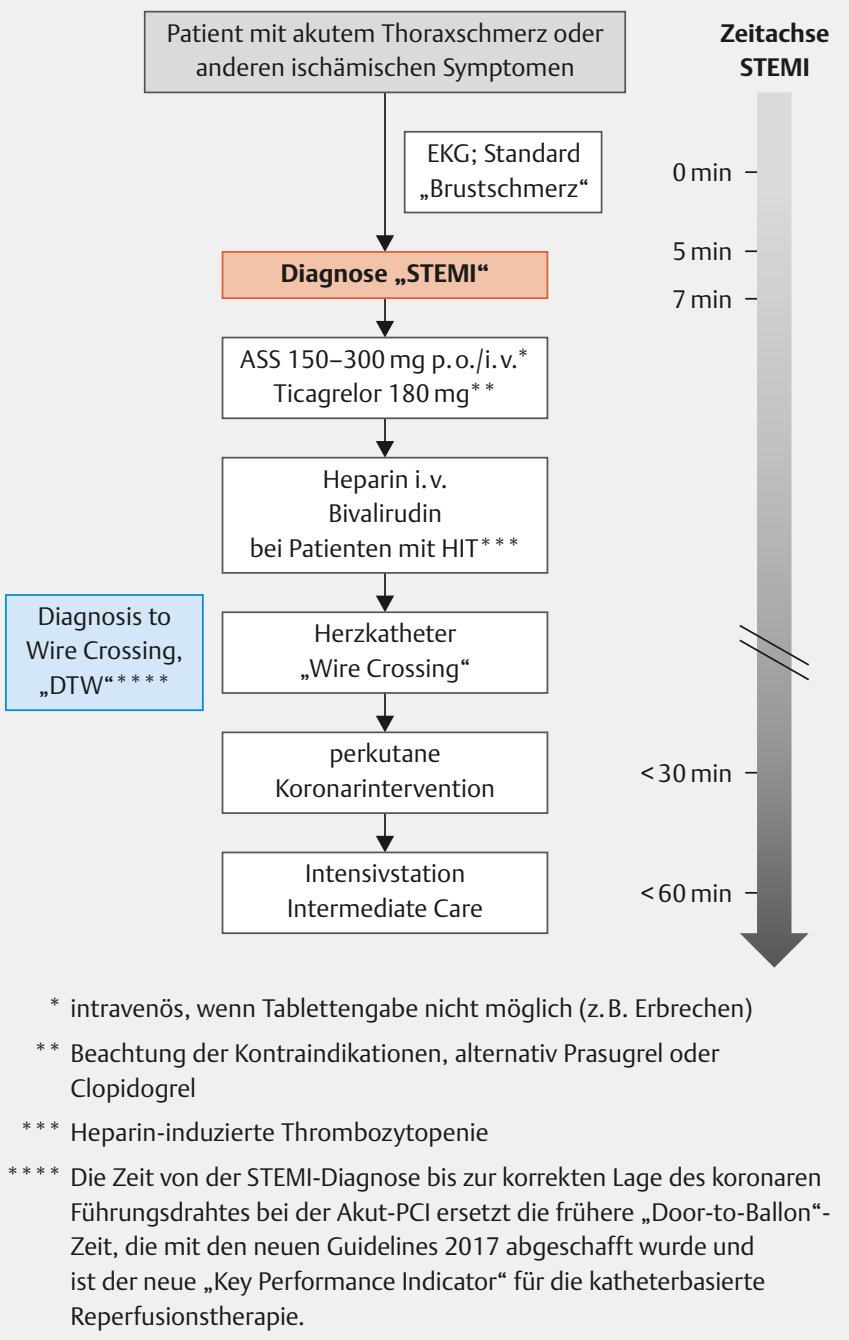

- Abb. 2 Beispiel eines Flowcharts zur Verwendung in einem Behandlungszentrum (hier: Notaufnahme Charité) mit Hinweisen zu weiteren Informationen und Ablaufplänen nach aktueller Anpassung an die neuen Guidelines der ESC [7].

\footnotetext{
Merke

Es ist wichtig, dass in einer regionalen Behandlungskette einheitlich vorgegangen wird und dass dieses sicher beherrscht wird. Die aktuelle medizinische Vorgehensweise beim akuten Brustschmerz findet sich bei Möckel u. Störk [10].
}

\section{Antithrombotisches Management: Hilfe durch Standards beim Einsatz neuer Therapien}

Allein im Bereich der antithrombotischen Therapie sind in den letzten Jahren neue Medikamente entwickelt und in internationalen Megatrials untersucht worden. Dabei gibt es mittlerweile konkurrierende Substanzen, die von den Leitlinien in ähnlicher Weise empfohlen werden (z. B. Prasugrel und Ticagrelor) und deren Einsatz eine klare Regelung erfordert. Dabei wird man sich typischerweise in einem Zentrum auf ein Vorgehen einigen und dies schriftlich in Form einer Ablaufanweisung formulieren.

In einer Publikation von Lickfeld u. Mitarb. [4] zum Thema der antithrombotischen Therapie beim Infarkt wird deutlich, dass sich das Vorgehen auch an den jeweiligen regionalen Gegebenheiten orientieren muss. Auch hier ist es hilfreich, wenn auf der übergeordneten Ebene der eEPK Konsens herrscht, z. B. wann ein moderner ADP-Rezeptor-Blocker ausgewählt und appliziert wird, um sich dann nur noch auf die konkrete Medikamentenauswahl konzentrieren zu können.

\section{Wenn es schnell gehen muss: Standards als Hilfen bei seltenen Zwischenfällen}

In kaum einer anderen Situation als bei der Komplikation im Herzkatheter sind Standards so wichtig wie hier. Da sie selten vorkommen, werden oft Details des Vorgehens nicht erinnert oder verschwimmen in der angespannten Situation. Dabei wird deutlich, dass Standards nicht nur vorhanden, sondern auch geübt und beherrscht werden müssen. In der Arbeit von Radke u. Mitarb. wird das Thema des standardisierten Komplikationsmanagements im Herzkatheterlabor ausführlich dargestellt [6]. Die Box „Beispiel einer SOP zum Vorgehen beim „No-Reflow“Phänomen“ zeigt das konkrete Beispiel einer SOP für die häufigste Komplikation im Katheterlabor, das „No-Reflow“-Phänomen. Diese SOP hilft, schnell, effektiv und richtig vorzugehen. 


\section{PRAXIS}

\section{Beispiel einer SOP zum Vorgehen}

beim „No-Reflow“-Phänomen [6]

Checkliste bei No-Reflow-Phänomen

- alternative Ursache prüfen (Luftembolisation, Thrombus, Spasmus, Dissektion)

- Sauerstoffgabe einleiten (2-4 I O $/$ /min)

- ACT prüfen (Ziel: 200-250 s)

- Nitrogabe (200 $\mu$ g i.c.)

- Volumengabe (z. B. Ringer-Lösung 500 ml i.v.)

- Adenosingabe: repetitive intrakoronare Boli von $20 \mu \mathrm{g}$ (RCA) und $30 \mu \mathrm{g}$ (LCA) bis zu $60 \mu \mathrm{g}(\triangleright$ Tab. 1)

- alternativ Verapamilgabe: Bolus max. $200 \mu \mathrm{g}$ i.v. Verapamil (Cave: Schrittmacher)

- alternativ Nitroprussidgabe: Bolus max. $200 \mu \mathrm{g}$ i. v.

\section{Ausblick und Schlussfolgerungen}

Standardisierte Vorgehensweisen sind in einer zunehmend prozessual aufgebauten medizinischen Versorgungskette unverzichtbar. Für das ACS sind die methodischen Hintergründe und Vorschläge zum Vorgehen publiziert worden. Zukünftig werden jedoch IT-basierte Hilfsmittel das Vorgehen am Patienten entscheidend unterstützen können. Dabei ist vorstellbar, dass die behandelnden Ärzte und ihre Teams mit mobilen Endgeräten (iPAD, Tablet-PC o.ä.) patientennah stets aktuelle Informationen zur Verfügung haben und ggf. auch wichtige einfache Dokumentationsschritte vornehmen können. So könnte das Behandlungsteam perspektivisch durch eine „CPU-App“ mit Informationen unterstützt und von der Dokumentation entlastet werden. Eine solche Applikation wird aktuell von der DGK durch unsere Task Force (siehe oben) entwickelt. Diese Entwicklung wird es dann gegen den aktuellen ungünstigen Trend (immer mehr Verwaltungsarbeit) in Zukunft möglich machen, dass sich jede Berufsgruppe und jeder Spezialist wieder vermehrt auf seine Kernkompetenz konzentriert und diese zum Nutzen des Patienten voll zur Wirkung bringen kann.

\section{Interessenkonflikt}

Der Autor gibt an, dass keine Interessenkonflikte vorliegen.
- Tab. 1 Herstellung Adenosin-Stammlösung/-intrakoronare Lösung.

\begin{tabular}{|c|c|}
\hline Lösung & Herstellung \\
\hline \multirow[t]{4}{*}{$\begin{array}{l}\text { Adenosin- } \\
\text { Stammlösung }\end{array}$} & 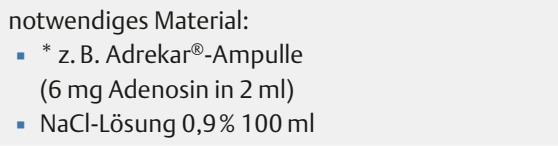 \\
\hline & $\begin{array}{l}\text { Abziehen und Verwerfen von } 40 \mathrm{ml} \text { aus der } \\
\mathrm{NaCl} \text {-Flasche }\end{array}$ \\
\hline & $\begin{array}{l}\text { Injektion der Adrekar }{ }^{\circledR} \text {-Ampulle in die verbliebenen } \\
60 \mathrm{ml} \mathrm{NaCl} \text {-Lösung }\end{array}$ \\
\hline & Durchmischen der $\mathrm{NaCl}$-Lösung \\
\hline \multirow{3}{*}{$\begin{array}{l}\text { Adenosin-Lösung } \\
\text { zur intrakoronaren } \\
\text { Injektion }(10 \mu \mathrm{g} / \mathrm{ml})\end{array}$} & $\begin{array}{l}\text { Abziehen von } 1 \mathrm{ml} \text { Stammlösung in eine sterile } \\
\text { 10-ml-Spritze }\end{array}$ \\
\hline & Auffüllen der 10-ml-Spritze mit $9 \mathrm{ml} \mathrm{NaCl} \mathrm{0,9 \%}$ \\
\hline & $\begin{array}{l}10 \mathrm{ml} \text { der Injektionslösung enthalten } 100 \mu \mathrm{g} \\
\text { Adenosin }(10 \mu \mathrm{g} / \mathrm{ml})\end{array}$ \\
\hline
\end{tabular}

Autorinnen/Autoren

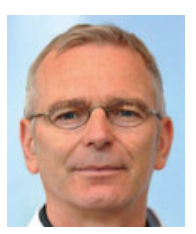

\section{Martin Möckel}

Univ.-Prof. Dr. Martin Möckel ist Ärztlicher Leiter Notfallmedizin/Rettungsstellen und Chest Pain Units CVK/CC an der Charité Universitätsmedizin Berlin.

Korrespondenzadresse

Univ.-Prof. Dr. Martin Möckel, FESC, FAHA

Charité-Universitätsmedizin Berlin

Campus Virchow-Klinikum und Mitte

Augustenburgerplatz 1

13353 Berlin

martin.moeckel@charite.de

\section{Literatur}

[1] Thygesen K, Alpert JS, Jaffe AS et al. The Writing Group on behalf of the Joint ESC/ACCF/AHA/WHF Task Force for the Universal Definition of Myocardial Infarction. Third universal definition of myocardial infarction. Eur Heart J 2012; 33: 25512567

[2] Vollert J, Hamm C, AG SOP Kardiologie, Möckel M. Prozessorientierte und standardisierte Umsetzung von DGK-Leitlinien: Vorgehen beim ST-Strecken-Hebungsinfarkt. Kardiologe 2009; 3: 388-405

[3] Möckel M, Vollert ], Hamm C, AG SOP Kardiologie. „Standard operating procedures" für den akuten ST-Streckenhebungsinfarkt: Anwendung prozessbasierter Grundsätze. Kardiologe 2010; 4: 124-134 
[4] Lickfeld T, Hamm C, Möckel M et al. Antithrombotische Therapie beim ST-Streckenhebungsinfarkt: Prozessorientierte und standardisierte Umsetzung von DGK-Leitlinien. Kardiologe 2010; 4: 107-123

[5] Moser M, Lickfeld T, Möckel M et al. „Standard operating procedures" zur Umsetzung der Leitlinien beim Nicht-ST-Hebungsinfarkt. Kardiologe 2010; 4: 389-399

[6] Radke PW, Wolfrum S, Elsässer A et al. „Standard operating procedures" für periprozedurale Komplikationen im Herzkatheterlabor. Koronardiagnostik und -therapie. Kardiologe 2011; 5: 27-37

[7] The Task Force on the management of ST-segment elevation acute myocardial infarction of the European Society of Cardiology (ESC). 2017 ESC Guidelines for the management of acute myocardial infarction in patients presenting with STsegment elevation. Eur Heart J 2018; 39: 139-177. doi:10.1093/eurheartj/ehx393
[8] Scholz KH, Maier SKG, Jung | et al. Reduction in Treatment Times Through Formalized Data Feedback: Results From a Prospective Multicenter Study of ST-Segment Elevation Myocardial Infarction. JACC Cardiovasc Interv 2012; 5: 848-857

[9] Thiele H, Zeymer U, Neumann F] et al. Intraaortic Balloon Support for Myocardial Infarction with Cardiogenic Shock. N Engl J Med 2012; 367: 1287-1296

[10] Möckel M, Störk T. Akuter Brustschmerz. Internist 2017; 58: 900-907. doi:10.1007/s00108-017-0299-8

\section{Bibliografie}

DOI https://doi.org/10.1055/s-0043-122084

Kardiologie up2date 2018; 14: 109-114

(c) Georg Thieme Verlag KG Stuttgart · New York ISSN 1611-6534 